\title{
The three-dimensional graphic method for quantifying body position
}

\author{
WILLIAM M. K. TROCHIM \\ Southern Connecticut State College. 501 Crescent Street. New Haven, Connecticut 06515
}

\begin{abstract}
The three-dimensional graphic method for quantifying body position is a series of observer procedures and computer programs designed to yield three-dimensional (height, width, and depth) coordinates for various body points. These coordinates can be graphed by computer in several different ways, and can be analyzed mathematically to provide information about a wide variety of variables, including interpersonal distance and body activity. The procedure for collecting and analyzing the data is explained and the computer programs developed for the method are described.
\end{abstract}

The quantitative measurement of body movements involves the assignment of a set of numerical values to the positions or movements being analyzed. For the most part, the quantitative methods reported (Ellis \& Pryer, 1959; Foshee, 1958; Jones \& Hanson, 1961: Jones \& Narva, 1955) are hampered by two major disadvantages. First, they often employ procedures and equipment that tend to foster reactive effects in the subjects being observed. Foshee (1958), for example, makes use of a desk or a chair which is secured on a platform supported at four comers by solid rubber stoppers. Jones and Narva (1955) required subjects to be seated in profile before a grid background and photographed. Jones and Hanson (1961) attached pieces of reflecting tape to "key" parts of the body, thus making body movements more visible on film. It is doubtful that subjects subjected to these procedures remain unaffected. Also. most of these quantitative methods are designed to measure only certain aspects of body movement (e.g.. activity, rate of movement, direction of movement, etc.). It would be impossible, in most cases, to modify a method designed to measure activity so that it also could measure body posture or interpersonal distance.

One quantitative method (Dierssen, Lorenc, \& Spitaleri, 1961) is not subject to these weaknesses. The method attempts to account for three aspects of body movement: movement occurring in space, movement occurring in a time period during which the successive positions change very rapidly, and movements resulting from the changing position of many parts of the body at the same time. To record movement, a transparent grid is attached to a movie screen and height and width coordinates are plotted for various points on the body. The method is nonreactive in that the procedure is carried out after the filming has taken place. It is capable of generating a great deal of information about a wide variety of variables involved in body movement, including activity, distance, and body angle or posture.

Presented in partial fulfillment of the requirements for the degree of Master of Arts, Southern Connecticut State College, New Haven, Connecticut, Dr. Robert Dowd, advisor.
The major weakness is that it provides only a twodimensional projection of a movement which is threedimensional.

\section{THE THREE-DIMENSIONAL GRAPHIC METHOD}

The three-dimensional (3-D) graphic method described in this paper consists of a series of observer procedures and computer programs which allow predictions concerning the depth dimension of body movement. The three-dimensional coordinates obtained are analyzed by computer to yield information about many body movement variables, including activity level and interpersonal distance.

\section{Method}

Procedure, After videotape records have been made, five steps must be carried out to collect the body position data to be analyzed by the computer. First, the lengths between chosen body points of a subject are determined with a tape measure. Using the 19 body points listed in Table 1, only 11 lengths between adjoining body parts (e.g., toe to heel, heel to knee etc.) need to be measured.

Second, the $0,0,0$ point or "origin" of a transparent grid is chosen. This grid, divided into equal (e.g., $1 / 2$ in.) units, is placed on the screen of the television monitor. The origin of the grid is determined arbitrarily, but it should be a point in the room that can be seen easily trom the videotape. The subject's body position is measured in relation to that point.

Third, the "depth entry point" or the depth of a specific part of the subject's body must be determined for every videotape (frame or film) analyzed. To do this, the observer uses a depth grid. This grid is constructed by marking off on a transparency the depth scale units from the origin point. The transparency is placed on the television monitor. If the grid is marked off in relation to the floor, there are four possible entrance points of the 19 body points listed in Table 1, the left and right toe and the left and right heel of the subject's feet. The depth entry point is determined once for every subject in each frame of film and simply requires the observer to place the depth grid on the screen and record the depth of any one of the four points that is touching the floor,. The observer must estimate the depth from the grid.

Fourth, the observer records the height and width coordinates for each body part, using the equal unit transparent grid. The points must be plotted in the specified order which the computer is programmed to follow. 
Table 1

Sample Output of Conversion Program for One Frame of Film

\begin{tabular}{lrrr}
\hline Frame 1 & Width & Height & Depth \\
\hline Left Toe & -7.00 & -8.00 & 14.37 \\
Left Heel & -11.00 & -8.00 & 10.76 \\
Left Knee & -5.00 & 5.00 & 18.47 \\
Center & -11.00 & 8.00 & 10.37 \\
Right Knee & -14.00 & 4.00 & 19.63 \\
Right Heel & -13.00 & -7.00 & 24.47 \\
Right Toe & -15.00 & -10.00 & 28.47 \\
Collar & -10.00 & 20.00 & 1.11 \\
Left Shoulder & -6.00 & 19.00 & -.67 \\
Left Elbow & -4.00 & 14.00 & 4.47 \\
Left Wrist & -7.00 & 13.00 & 8.99 \\
Right Shoulder & -14.00 & 19.00 & -.67 \\
Right Elbow & -17.00 & 15.00 & 4.84 \\
Right Wrist & -17.00 & 13.00 & 9.99 \\
Chin & -10.50 & 21.00 & 3.28 \\
Tip of Nose & -10.50 & 22.50 & 3.63 \\
Hairline & -11.00 & 26.00 & .95 \\
Left Ear & -8.00 & 23.00 & 2.15 \\
Right Ear & -12.00 & 23.00 & 1.14 \\
\hline
\end{tabular}

Finally. directional indicators for each body point must be determined in specified order beginning with the depth entry point. This involves a simple discrimination concerning which point (of two) is closer to the camera. The closest point is scored as +1.00 , and the farthest as -1.00 . The directional indicator tells the computer whether to add or to subtract the calculated depth quantity from the previous point's depth coordinate.

When the data have been collected in this manner, the depth of all the body points may be calculated in succession using the following formula:

Given: Point $A=\left(A_{1}, A_{2}, A_{3}\right)$ where $A_{1}$ and $A_{2}$ are the height and width coordinates for Point $A$ and $A_{3}$ is depth entry point:

Point $B=\left(B_{1}, B_{2}, B_{3}\right)$ where $B_{1}$ and $B_{2}$ are the height and width coordinates for Point $B$ and $B_{3}$ is depth point desired:

$\mathbf{L}=$ body length between $\mathrm{A}$ and $\mathrm{B}$ :

$\mathrm{X}=$ directional indicator.

Then:

$$
B_{3}=X \sqrt{L^{2}-\left(A_{1}-B_{1}\right)^{2}-\left(A_{2}-B_{2}\right)^{2}}+A_{3} .
$$

A computer program, written in FORTRAN. applies the formula successively on pairs of points in the specified order. ${ }^{1}$ A sample of the output of this conversion program is shown in Table 1 . The 19 body points currently used are listed first, followed by the height and width coordinates which are plotted by the cbserver. The third column shows a list of the depth coordinates for each of the 19 body points as calculated by the conversion program.

Reliability and Validity. The validity and reliability of the 3-D graphic method were determined. Two subjects (a male and a female) were each instructed to assume five different seated positions. I he observers measured the depths of each subject's body points in each position and, after the videotaping session. measured body lengths. Ten untrained observers then used the 3-D graphic method to record the 10 body positions.

The Pearson product-moment correlations between the body length measurements made by the two observers were .986 and 955. The reliability of the depth calculations was estimated by computing the Pearson product-moment correlations between pairs of observers icross the 19 body points. These coefficients ranged from .99 to 38 with a median of .93 . The low correla- tions were primarily due to three observers who were less consistent than the other seven. Of the 450 correlations. 329 or $73 \%$ were above .80 . The validity of the depth calculations yielded by the conversion program was assessed by computing the correlations between these values and the depths of the same body points measured by hand. These coefficients ranged from .99 to -.26 with a median correlation of .93 The low correlations again can be attributed to a few observers. The least consistent observer's coefficients ranged from .95 to -.02 with a median of .67 , the most consistently accurate observer had validity coefficients which ranged from .99 to .62 and had a median of 94 .

\section{ANALYSES}

The major advantage of the 3-D graphic method is its ability to yield information about a wide variety of variables involved with body movement and position. There are generally two types of analyses that can be performed on the three-dimensional coordinates given by the conversion program. The data can be represented graphically. Because the computer stores all three coordinates for each body point, it can be programmed to print out a picture of the body positions from any angle. Also, the data can be analyzed mathematically to yield information about distances between body points and the angles which they form.

Two graphing programs have been used in conjunction with the 3-D graphic method. Both are written in the FORTRAN language and graph a front, top, and side view of the subjects videotaped. They differ primarily in the output devices used to print the graphs. One program uses a standard printer which graphs in a "type.

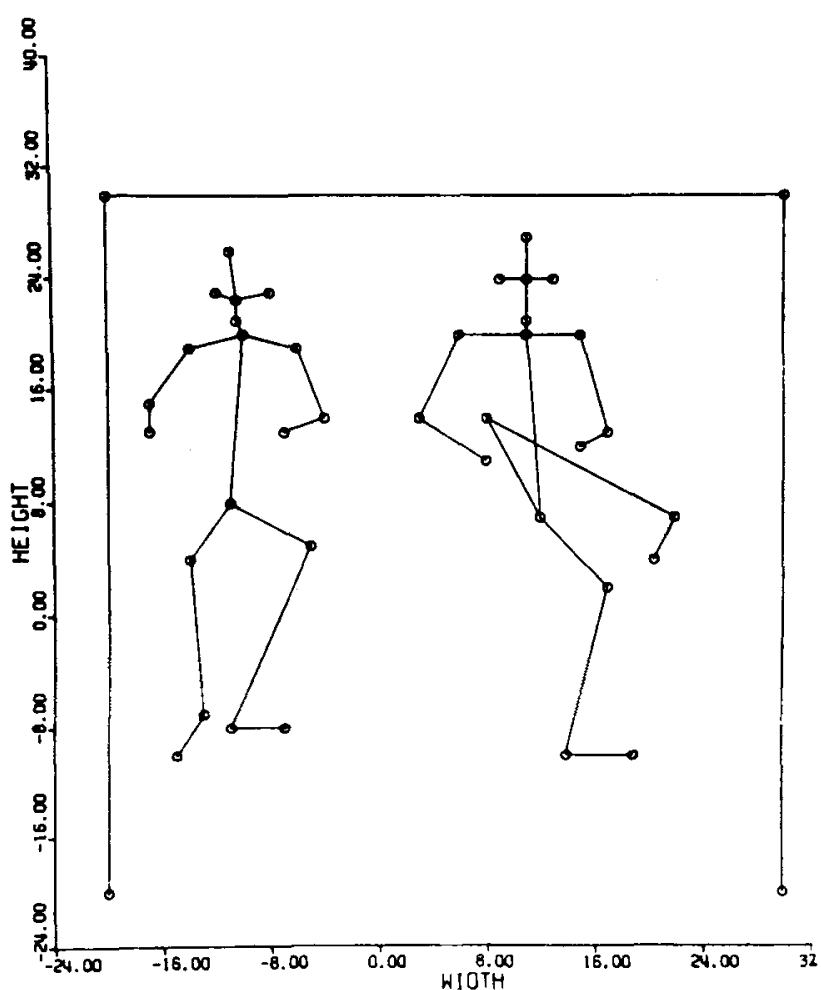

Figure 1. Front view Cal-Comp graph of two subjects. 


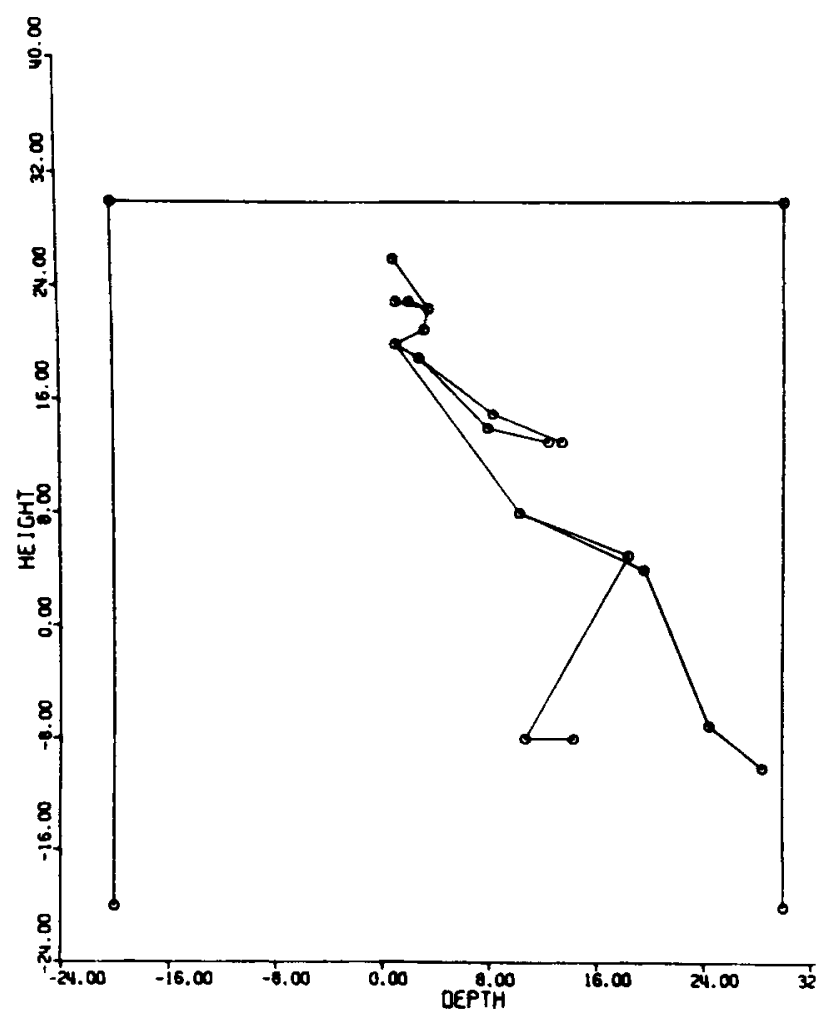

Figure 2. Side view Cal-Comp graph of Subject 1.

writer" mode; the program prints only integer values, thus causing some distortion in the graph. The second

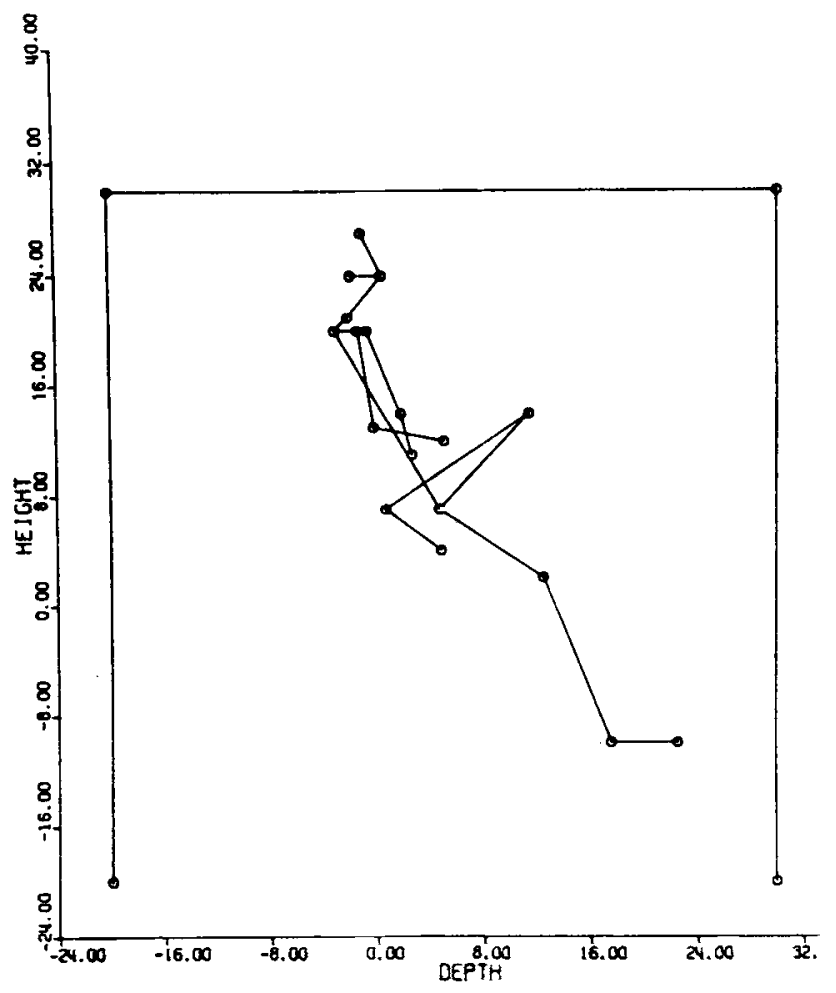

Figure 3. Side view Cal-Comp graph of Subject 2.

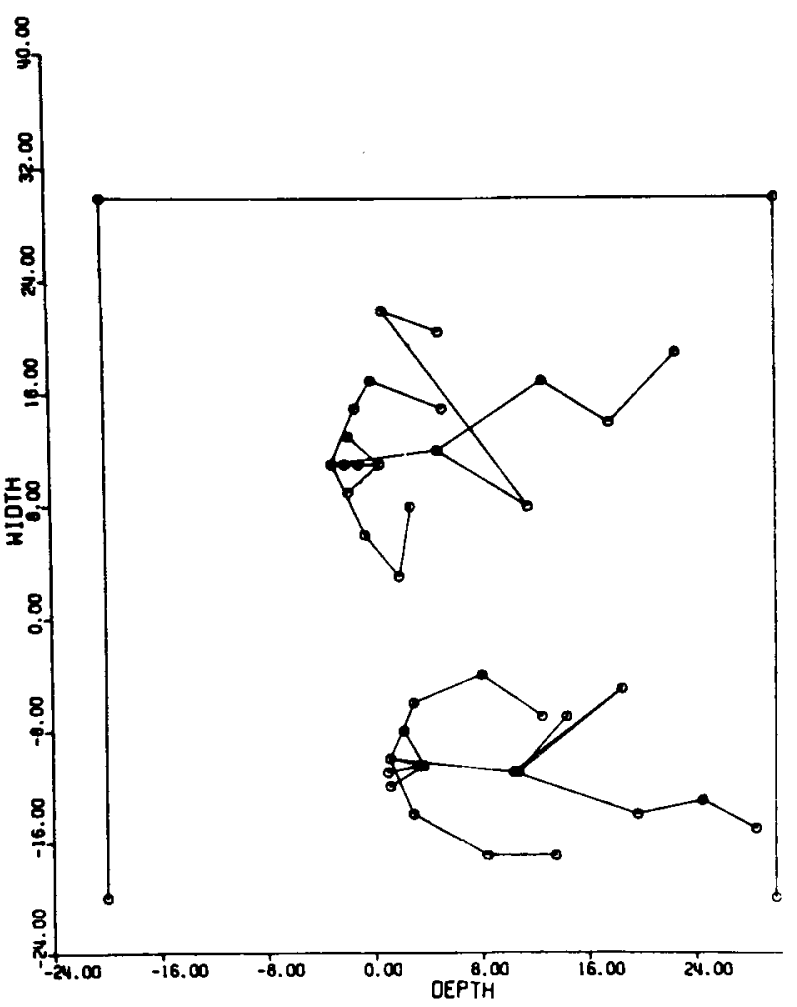

Figure 4. Top view Cal-Comp graph of two subjects.

graphing program makes use of a Cal-Comp plotting device to draw the graphs. The Cal-Comp plotter gives a more pleasing and exact representation of the body positions than does the standard printer, but the product is slightly more expensive, a consideration when many graphs are needed. Figure 1 is a Cal-Comp graph of two subjects viewed from the front. This view represents the videotaped view because it relies only on the height and width measurements of the observer. Figures 2 and 3 are side views of the same two subjects separately, and Figure 4 is the top view. The side and top views are necessarily based upon the depths calculated by the conversion program and therefore give visual testimony to the accuracy of the method.

The graphing programs have two major uses. First, the graphs are useful for their ability to portray various body positions. The graphs also can provide important feedback to the observer about his accuracy in using the 3-D graphic method. By using an on-line computer in conjunction with a cathode ray tube terminal, it is possible for the observer to correct his observations immediately until his conception of the videotaped position "matches" the graphic representation.

The interpersonal distance program calculates the distances and mean distances between the corresponding body points of two subjects. Table 2 shows a sample of the interpersonal distance program output for a few of the body points in a single frame of film.

A summary listing of the mean distances for each 
Table 2

Sample Output of Interpersonal Distance and Body Activity Level Programs

Interpersonal Distance for Frame 1

$\begin{array}{ll}\text { Left Toe } & 27.43 \\ \text { Left Heel } & 27.06 \\ \text { Left Knee } & 22.00 \\ \text { Center } & 24.05 \\ \text { Mean } & 24.05\end{array}$

Mean Interpersonal Distances for Five Frames

$\begin{array}{ll}\text { Left Toe } & 30.84 \\ \text { Left Heel } & 28.69 \\ \text { Left Knee } & 24.51 \\ \text { Center } & 25.16 \\ \text { Grand Mean } & 26.14\end{array}$

Body Activity Levels from Frame 1

$\begin{array}{lrr}\text { Left Toe } & 6.07 & 8.91 \\ \text { Left Heel } & 5.50 & 7.84 \\ \text { Left Knee } & 7.96 & 10.54 \\ \text { Center } & 8.43 & 10.82 \\ \text { Mean } & 8.17 & 13.96\end{array}$

Mean Body Activity Levels for Four Frames

$\begin{array}{lrr}\text { Left Toe } & 13.20 & 16.45 \\ \text { Left Heel } & 10.99 & 16.89 \\ \text { Left Knee } & 9.41 & 12.82 \\ \text { Center } & 9.90 & 7.67 \\ \text { Grand Mean } & 12.53 & 14.06\end{array}$

body part over $x$ number of frames is shown at the end of the output. The grand mean is simply the mean of each frame's mean for $x$ number of frames.

The body activity level program computes, for parts of the body (e.g., right wrist) and the whole body, the activity level over a period of time by calculating the distance between one frame of film and the next. Table 2 shows a sample of the output for a few of the body points for two subjects. As with the interpersonal distance program, a summary listing for $\mathrm{x}$ number of frames is also provided.

Other analyses can be conducted. Currently, programs are being written in FORTRAN to analyze the sociofugal-sociopetal orientation of Hall's (1963) proxemic notation system and such variables as forward lean and shoulder orientation (Mehrabian, 1967). It is possible to perform analyses on any position or movement variables for which the relationship between body parts can be stated mathematically. The programs can also be used with other data collection systems. For example, the three-dimensional coordinates can be collected by using two cameras, one providing a view from the front and one from the side. Once these coordinates are determined, the analyses can proceed as in the 3-D graphic method. A two-canera system requires more analysis time.

The process of collecting the data from the videotape picture can be accelerated by utilizing a light pen for storing the coordinates presented on a display. Methods for coding and storing the videotape can also be developed. The equipment already exists (Sackett, Stephenson, \& Ruppenthal, 1973), and the beginnings of such a system were developed several years ago by Ekman and Freisen (1969).

\section{REFERENCES}

Dierssen, A., Lorenc, M., \& Spitaleri, R. A new method for graphic study of human movements. Neurology, 1961, 2, 610-618.

Ekman, P., \& Freisen, W. A tool for the analysis of motion picture film or videotape. American Psychologist, 1969, 24, 240-243.

Ellis, N., \& Pryer, R. Quantification of gross bodily activity in children with severe neuropathology. American Journal of Mental Deficiency, 1959,63, 1034-1037.

Foshee, J. Studies in activity level: I. Simple and complex task performance in defectives. American Journal of Mental Deficiency, $1968,62,883-885$.

Hall, E. T. A system for the notation of proxemic behavior. American Anthropologist, 1963,65, 1003-1026.

Jones, F.. \& Hanson, $J$. Time-space pattern in a gross body movement. Perceptual and Motor Skills, 1961, 12, 35-41.

Jones, F., \& Narva, M. Interrupted light photography to record the effect of changes in the poise of the head upon patterns of movement and posture in man. Journal of Psychology. $1955,40,125,-131$

Mehrabian, A. Orientation behaviors and non-verbal attitude communication. Journal of Communication, 1967, 17. 324-332.

Sackett, G., Stephenson, E., \& Ruppenthal, G. Digital data acquisition systems for observing behavior in laboratory and field settings Behavior Research Methods \& Instrumentation, $1973,5,344-348$

\section{NOTE}

1. All computer programs are available from the author upon request. 